Brown, G. D. \& Rainbow, C. (1956). J. gen. Microbiol. 15, 61-69

\title{
Nutritional Patterns in Acetic Acid Bacteria
}

\author{
By G. D. BROWN aNd C. RAINBOW \\ Department of Applied Biochemistry, University of Birmingham
}

SUMMARY: The nutritional patterns of 28 typed and untyped strains of Acetobacter spp. ranged between extremes with predominant lactate (lactaphilic) or glucose (glycophilic) metabolism. Typical lactaphilic strains did not require exogenous supplies of growth factors and gave rise to new ninhydrin-reacting substances when cell suspensions were incubated in solutions of proline, glutamate and aspartate. Under similar conditions, glycophilic strains yielded no new ninhydrin-reacting substances and required added nicotinate, pantothenate and, in some cases, $p$-aminobenzoate for growth.

All strains utilized ammonium sulphate as sole source of nitrogen for growth. Under such circumstances, most lactaphilic strains grew readily when lactate provided the only source of energy and carbon: other organic acids, even to be partially effective as substitutes for lactate, required the simultaneous presence of glucose. Glycophilic strains utilized ammonium sulphate as sole source of nitrogen forgrowth slowly and to a limited extent in glucose medium, the degree of growth being increased by the presence of certain organic acids. Characteristically, glycophilic strains required certain sugars or sugar alcohols for growth, but were little or not at all stimulated by lactate.

Working with 7 strains of acetic acid bacteria, Rainbow \& Mitson (1953) distinguished organisms having either a predominant lactate metabolism (the Acetobacter mobile group) or a predominant glucose metabolism (the $A$. viscosum group). In this work, we prefer to refer to the former as the 'lactaphilic' and to the latter as the 'glycophilic' group. The validity of the distinction between these groups has now been extended by tests on a further 21 strains of Acetobacterspp. The distinction is shown to be associated, circumstantially at least, with differences in respect of requirements for exogenous growth factors and in behaviour of cell suspensions in solutions of amino acids biochemically related to L-glutamic acid.

\section{METHODS}

Test organisms and inocula. The organisms used in this work were the strains of Acetobacter used by Rainbow \& Mitson (1953) and the following: Acetobacter aceti (NCTC 6428), A. acetosum (NCTC 2224), A. ascendens (NCTC 4937), $A$. orleanense (NRRL B 55), $A$. oxydans (NCTC 6720), A. rancens (NRRL B 65), $A$ suboxydans (NCTC 6430), $A$. suboxydans (NCTC 7118) and the untyped strains D1, D2, D3, D4, D5, D6, D12, G1, A1, A2, A8, A4 and A5. The unclassified strains were isolated frombeer or brewers' pitching yeasts by plating on malt agar under aerobic conditions. Each undetermined isolate produced a surface film or pellicle when grown on liquid media and consisted of catalasepositive, Gram-negative rods which oxidized ethanol to acetic acid during growth in $10 \%$ malt extract containing $2 \%(v / v)$ ethanol. Stock cultures 
were maintained and inocula for growth tests prepared according to Rainbow \& Mitson (1953) with the modification that certain inocula, treated similarly after harvesting, were grown in clarified $10 \%$ malt extract. For work with suspensions, organisms were grown in $6 \mathrm{ml}$. portions of medium for 2-4 days, and the entire subculture transferred to 60-100 ml. of medium I contained in a conical flask ( $250 \mathrm{ml}$. capacity). After incubation at $28^{\circ}$ for $6-7$ days, the organisms were harvested (centrifuge), washed twice with sterile $0.85 \%(\mathrm{w} / \mathrm{v}) \mathrm{NaCl}$ solution and resuspended in 30-50 ml. of the same solution, aseptic conditions being observed throughout.

Media. (a) Clarified malt extract was prepared by autoclaving (15 lb./sq.in. for $7 \mathrm{~min}$.) a $10 \%(\mathrm{w} / \mathrm{v})$ solution of dried malt extract (Muntona Ltd., Bedford) with $c .0 .5 \mathrm{~g}$. Celite filter-aid $/ 100 \mathrm{ml}$. of extract. This mixture was stored in the refrigerator for 3 days and then filtered through Whatman no. 42 paper.

(b) Medium I was the basal medium $a$ described by Rainbow \& Mitson (1953) but for tests involving glycophilic strains lactic acid was omitted and $1.0 \mathrm{mg}$. each of adenine and uracil/100 ml. were added. Media II, III and IV were identical with I, except that constituents were omitted as follows: glucose (from II), casein acid hydrolysate (CAH; from III) and glucose and CAH (from IV). In addition, the concentration of ammonium sulphate in media III and IV was increased to $0 \cdot 3 \mathrm{~g} . / 100 \mathrm{ml}$.

Materials. Sugars, sugar alcohols and organic acids were of Laboratory Reagent grade excepting the following, which were of A.R. grade: citric, lactic and succinic acids, and sodium acetate $\left(3 \mathrm{H}_{2} \mathrm{O}\right)$ which was used for all experiments involving acetate. The amino acids were commercial preparations, the purity of which was examined by paper chromatography. The L-proline invariably contained a trace of hydroxyproline and the DL-alanine invariably contained glycine, from which it was partially separated by recrystallization from aqueous acetone. Occasionally, samples of L-glutamic acid and L-arginine contained traces of aspartic acid and ornithine respectively. The presence of all such impurities was allowed for in interpreting the results obtained with suspensions of organisms.

The CAH used in media was the 'vitamin-free' sample (Allen and Hanburys Ltd.) reported on by Moore \& Rainbow (1955).

Growth tests were carried out as described by Rainbow \& Mitson (1953). In a few cases, when strains A 1, A 2, A 3, A4, A5 and G 1 were grown on certain substrates, the organisms formed coherent pellicles at the surface, so that only a crude visual assessment of growth was possible (e.g. in Table 1). Growth tests were allowed to proceed for 7 days, Spekker turbidity readings being taken after $48 \mathrm{hr}$. and thereafter daily. Turbidity readings reported in this paper refer to measurements made after 7 days unless otherwise stated. However, such readings sometimes conceal certain kinetic aspects of growth, particularly with respect to growth on media supplemented with organic acids. For example, certain strains (Table 1) which grew on acetate or ethanol did so only after a considerable lag and Acetobacter mobile grew on glucose +lactate (Table 2) only after 6 days of incubation. 
The $\mathrm{pH}$ values of the culture fluids of all tubes in growth tests were determined colorimetrically after 7 days.

Experiments with suspensions of organisms were carried out at $\mathbf{2 8}^{\circ}$ and initial pH 5.8 in systems consisting of $3 \mathrm{ml}$. of substrate solution $+3 \mathrm{ml}$. of suspension. The latter was prepared as above and, when necessary, previously diluted with further sterile saline so that the final reaction mixture had an

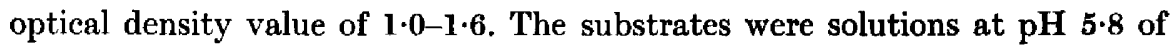
$0.6 \%(\mathrm{w} / \mathrm{v})$ of the $\mathrm{L}$ or $1.2 \%(\mathrm{w} / \mathrm{v})$ of the $\mathrm{DL}$ forms of the following amino acids: L-proline, L-glutamic acid hydrochloride, L-aspartic acid, DL-alanine and L-arginine hydrochloride. In some experiments, the amino acid solution was supplemented with glucose and/or potassium lactate (pH 5.8) added in the form of two drops $(c .0 .05 \mathrm{ml}$.) of sterile $50 \%(\mathrm{w} / \mathrm{v})$ solutions $/ 6 \mathrm{ml}$. test solution. Each experiment included the appropriate uninoculated blanks and a blank consisting of $3 \mathrm{ml}$. of a suspension of the test organism $+3 \mathrm{ml}$. of sterile $0.85 \%(\mathrm{w} / \mathrm{v})$ saline. Samples $(10 \mu \mathrm{l}$.) of supernatant fluid were withdrawn after $48 \mathrm{hr}$. (and when necessary at subsequent daily intervals up to 7 days), and examined by paper chromatography for content of amino acids as described by Chamberlain \& Rainbow (1954). The pH values of the culture fluids were determined colorimetrically at the end of the incubation period.

\section{RESULTS}

\section{Carbon and energy requirements}

The ability of certain sugars, sugar alcohols and organic acids to promote the growth of the test organisms was determined by growth tests in media II, III and IV. Solutions of test substances were adjusted, when necessary, to pH 5.8 with KOH solution before addition to the test medium. Tests were carried out in these media in order to obtain information concerning the utilization of the test substances as sources of $(a)$ energy (in medium II); $(b)$ carbon fragments (in medium III) and (c) energy and carbon fragments (in medium IV).

The results showed that each test strain fitted into one or other of the two groups distinguished by Rainbow \& Mitson (1953) and are illustrated (Table 1) by data for three strains selected as representative of all the strains tested.

The lactaphilic group comprised the following strains: Acetobacter aceti, $A$. acetosum, $A$. ascendens, $A$. orleanense, $A$. oxydans, $A$. rancens, $A$. suboxydans 6430, A. suboxydans 7113 and strains D1, D 2, D3, D4, D 5, D6, D 12 and G1. To these should be added Rainbow \& Mitson's $A$. mobile group ( $A$. acidummucosum, $A$. mobile and $A$. suboxydans 7069), which they closely resembled. They showed the following general features.

(a) In medium II, growth was stimulated in all cases by lactate, acetate or ethanol, lactate usually being the most effective. The sugars or sugar alcohols were completely or relatively ineffective or, like glycerol, effective only for a few strains. Growth was also stimulated to a small extent by organic acids other than lactate and acetate. The acids effective in this respect differed with the strain, succinate being the most often, and citrate the least often, effective. 
Since medium II contained potential fragments for biosynthesis (as CAH) those substances which promoted growth were likely to do so because they provided a suitable source of energy. This, however, is not necessarily so, as was shown by the fact that some growth took place in unsupplemented medium II, which was therefore itself not devoid of an energy-yielding material.

\section{Table 1. Sugars, sugar alcohols and organic acids as sources of carbon and energy for Acetobacter strains}

Growth tests were made at $2^{\circ}$ in medium I less lactate and glucose (medium II), less lactate and CAH (medium III) and less lactate, glucose and CAH (medium IV). Glucose was tested at $2 \%$ concentration and other substrates equimolar to it. Growth is recorded as excess optical density value over that of the appropriate blank: $0=<0.20 ; 1=0.21-0.50$; $2=0.51-0.80 ; 8=>80 ;+=$ growth observed to have occurred, but no quantitative measurement was possible.

\begin{tabular}{|c|c|c|c|c|c|c|c|c|c|}
\hline \multirow[b]{4}{*}{ Substrate } & \multicolumn{9}{|c|}{ Strain of Acetobacter } \\
\hline & \multicolumn{3}{|c|}{ D3 (lactaphilic) } & \multicolumn{3}{|c|}{$\begin{array}{l}\text { A. ascendens } \\
\text { (lactaphilic) }\end{array}$} & \multicolumn{3}{|c|}{ A 1 (glycophilic) } \\
\hline & \multicolumn{9}{|c|}{ Growth in medium } \\
\hline & II & III & $\mathbf{I V}$ & II & III & IV & II & III & IV \\
\hline Glucose & $\mathbf{0}$ & & 0 & 0 & $\mathbf{0}$ & $\mathbf{0}$ & 3 & & $\mathbf{1}$ \\
\hline Fructose & $\mathbf{0}$ & 0 & $\mathbf{0}$ & $\mathbf{0}$ & 0 & $\mathbf{0}$ & + & $\mathbf{0}$ & 2 \\
\hline Mannitol & $\mathbf{0}$ & $\mathbf{0}$ & 0 & o & 0 & $\mathbf{0}$ & + & 0 & 2 \\
\hline Sorbitol & o & 0 & 0 & $\mathbf{0}$ & $\mathbf{0}$ & $\mathbf{0}$ & + & $\mathbf{0}$ & $\mathbf{2}$ \\
\hline Glycerol & 1 & $\mathbf{0}$ & $\mathbf{0}$ & $\mathbf{0}$ & $\mathbf{0}$ & 0 & + & $\mathbf{0}$ & 2 \\
\hline Ethanol & 2 & 0 & 0 & 2 & o & o & + & $\mathbf{0}$ & 0 \\
\hline Acetate & $\mathbf{2}$ & $\mathbf{0}$ & $\mathbf{0}$ & 1 & $\mathbf{0}$ & $\mathbf{0}$ & $\mathbf{0}$ & $\mathbf{0}$ & 0 \\
\hline Citrate & $\mathbf{0}$ & $\mathbf{0}$ & 0 & $\mathbf{0}$ & $\mathbf{0}$ & $\mathbf{0}$ & 0 & 2 & 0 \\
\hline Fumarate & $\mathbf{0}$ & $\mathbf{0}$ & $\mathbf{0}$ & $\mathbf{0}$ & $\mathbf{0}$ & $\mathbf{0}$ & $\mathbf{0}$ & 1 & 0 \\
\hline$\alpha$-Ketoglutarate & $\mathbf{0}$ & $\mathbf{1}$ & $\mathbf{0}$ & 1 & o & $\mathbf{0}$ & 0 & $\mathbf{1}$ & 0 \\
\hline Lactate & $\mathbf{3}$ & 3 & $\mathbf{2}$ & $\mathbf{3}$ & $\mathbf{0}$ & $\mathbf{0}$ & 1 & 1 & 0 \\
\hline Malate & 0 & 1 & 0 & $\mathbf{1}$ & $\mathbf{0}$ & $\mathbf{0}$ & o & $\mathbf{1}$ & 0 \\
\hline Succinate & 1 & $\mathbf{3}$ & 0 & 1 & 0 & $\mathbf{0}$ & 0 & 0 & 0 \\
\hline $\begin{array}{l}\text { Growth of blank } \\
\text { (optical density } \\
\text { value) }\end{array}$ & 0.41 & 0.01 & 0.01 & $0 \cdot 29$ & 0.02 & 0.01 & 0.21 & $0 \cdot 57$ & $0 \cdot 01$ \\
\hline
\end{tabular}

(b) In medium III, which contained a potential source of energy (glucose) and ammonium sulphate as sole source of nitrogen, lactate was again the best substrate for growth, except for Acetobacter ascendens and $A$. oxydans (see under $(c)$ below). Other organic acids (but never acetate) also stimulated growth, succinate being the most effective, as well as being effective for the greatest number of strains (13). The other acids usually gave smaller stimulations and were effective for fewer strains as follows: $\alpha$-ketoglutarate (12), malate (10), citrate (4) and fumarate (2). The number, and frequently the magnitude, of the stimulatory effects promoted by organic acids, other than lactate and acetate, in medium III were greater than those promoted by the same acids in medium II. This suggests that these acids were more generally utilized as sources of carbon, rather than as sources of energy, by the lactaphilic strains. On the other hand, acetate and ethanol appeared to be sources 
of energy but not of carbon. The sugars and sugar alcohols were also ineffective as sources of carbon.

(c) In medium IV, only lactate supported growth. Thus, only lactate was available as a source of both energy and carbon for the lactaphilic strains. Acetobacter ascendens, $A$. oxydans and strain $\mathrm{D} 2$ were, however, exceptional and failed to grow in medium IV, no matter what supplement was added. It appears that, to obtain growth of $\boldsymbol{A}$. ascendens and $\boldsymbol{A}$. oxydans in a medium containing ammonia as sole source of nitrogen, binary mixtures of carbon compounds must be supplied (see below). However, it seemed reasonable to group these strains with the lactaphiles because they utilized lactate readily in medium II, and also because they grew in the absence of added growth factors (see below).

The glycophilic group comprises strains A 1, A2, A3, A 4 and A5. In medium II, best growth was obtained on glucose, mannitol, sorbitol and glycerol: fructose was also readily utilized for growth in most cases. In contrast to that of the lactaphilic group, growth of the glycophilic group in media II and IV was little, if at all, stimulated by lactate. In the presence of glucose but absence of a major source of organic nitrogen (medium III), only A 1 and A2 grew at all well, and growth was stimulated to some extent by citrate, fumarate, $\alpha$-ketoglutarate, lactate or malate, but not by acetate or succinate. Strains A3, A4, and A5 were scarcely or not at all stimulated by organic acids in any of the media: they grew rapidly and well only in presence of CAH. With these glycophilic strains should be grouped Acetobacter capsulatum, A. gluconicum, A. turbidans and $A$. viscosum which comprise Rainbow \& Mitson's $A$. viscosum group and which most closely resembled A1 and A2 in their response to organic acids; in addition, $A$. turbidans and $A$. viscosum were stimulated by succinate in medium III.

Binary mixtures as sources of energy and carbon. Whilst lactate was a source of energy and carbon for most lactaphilic strains, there were indications, described above, that the other organic acids provided either energy or carbon, but not both, for many of the test organisms. This possibility was tested further by growth tests in medium IV, in which all possible pairs of the following substrates were presented to the test organisms: glucose, glycerol, ethanol, acetate, citrate, fumarate, $\alpha$-ketoglutarate, lactate, malate, succinate. For these tests Acetobacter ascendens, A. oxydans, and Rainbow \& Mitson's 7 strains were used.

The results (examples in Table 2) showed that: $(a)$ binary mixtures which promoted the growth of the lactaphilic strains Acetobacter acidum-mucosum, $A$. mobile and $\boldsymbol{A}$. suboxydans (NCTC 7069) contained either citrate, fumarate, malate or succinate (but not $\alpha$-ketoglutarate) as one substrate (presumably providing carbon fragments) and either glucose, glycerol, ethanol or acetate as the other. Since $A$. acidum-mucosum and $A$. suboxydans NCTC 7069 grew well on lactate as sole source of carbon and energy, binary mixtures containing lactate also supported good growth. However, for growth of $A$. mobile on lactate in medium IV, a second substrate (glucose or glycerol) was required; growth then occurred after a considerable lag. The nature of these second 
substrates implied that lactate provided a source of carbon. Lactate may also provide a source of energy for $A$. mobile: thus it grows well in medium II containing lactate (Rainbow \& Mitson, 1953).

Not all pairs of the above substrates gave growth: thus, the growth of Acetobacter acidum-mucosum was not promoted by glucose or by glycerol, nor that of $A$. mobile by ethanol, no matter what other substrate was also present. Utilization of acetate for growth was observed only with $A$. acidum-mucosum when malate was the second substrate and, after a lag period of 6 days, with $A$. suboxydans when glucose was present.

\section{Table 2. Utilization of ammonia for growth of Acetobacter strains in presence of binary mixtures of organic compounds}

Growth tests were made at $28^{\circ}$ for 7 days in medium IV. $\mathbf{G}=$ glucose, $\mathbf{T}=$ glycerol, $\mathbf{E}=$ ethanol, $\mathbf{A}=$ acetate, $\mathbf{C}=$ citrate, $\mathbf{F}=$ fumarate, $\mathbf{K}=\alpha$-ketoglutarate, $\mathbf{L}=$ lactate, $M=$ malate, $\mathrm{S}=$ succinate. For other details, see legend to Table 1 . Mixtures not included below failed to promote growth significantly greater than the appropriate blanks.

\begin{tabular}{|c|c|c|c|c|c|c|c|}
\hline \multicolumn{8}{|c|}{ Strain } \\
\hline \multicolumn{2}{|c|}{ A. acidum-mucosum } & \multicolumn{2}{|c|}{ A. ascendens } & \multicolumn{2}{|c|}{ A. mobile } & \multicolumn{2}{|c|}{ A. capsulatum } \\
\hline Mixture & $\begin{array}{c}\text { Extent of } \\
\text { growth }\end{array}$ & Mixture & $\begin{array}{c}\text { Extent of } \\
\text { growth }\end{array}$ & Mixture & $\begin{array}{c}\text { Extent of } \\
\text { growth }\end{array}$ & Mixture & $\begin{array}{c}\text { Extent of } \\
\text { growth }\end{array}$ \\
\hline $\mathbf{A M}$ & 2 & $\mathbf{A M}$ & $\mathbf{2}$ & GM & 1 & GM & $\mathbf{1}$ \\
\hline $\mathbf{E M}$ & $\mathbf{3}$ & $\mathbf{E M}$ & $\mathbf{3}$ & GS & $\mathbf{1}$ & GF & 1 \\
\hline ES & 8 & LS & 8 & GL & 1 & GC & $\mathbf{1}$ \\
\hline $\mathbf{E F}$ & 3 & $\mathbf{L M}$ & 2 & $\mathbf{T M}$ & 1 & GK & 2 \\
\hline $\mathbf{L}+$ any & & LF & 2 & TS & $\mathbf{1}$ & TL & 2 \\
\hline other & 8 & LK & $\mathbf{8}$ & TL & 2 & $\mathbf{T F}$ & 2 \\
\hline substrate & & & & TF & 1 & TK & $\mathbf{3}$ \\
\hline
\end{tabular}

(b) Most binary mixtures which promoted the growth of Acetobacter ascendens and $A$. oxydans contained lactate (presumably energy-yielding) as one component and either $\alpha$-ketoglutarate, malate, succinate or (for $A$. ascendens only) fumarate. These strains resembled $A$. acidum-mucosum in that they grew on ethanol + malate mixtures and failed to utilize glucose for growth.

(c) Binary mixtures promoting the growth of the glycophilic strains (Acetobacter capsulatum, $A$. gluconicum, $A$. turbidans and $A$. viscosum) always contained glucose or glycerol as one, presumably energy-yielding substrate and, except for $\boldsymbol{A}$. capsulatum, glucose + glycerol also stimulated growth to a small extent. The other substrates complementary to glucose or glycerol were organic acids (never acetate) of which $\alpha$-ketoglutarate was particularly effective.

Variations of $\mathrm{pH}$ value during growth. Since media II, III and IV were not well buffered over the range $\mathrm{pH} 4-6$, the possibility was considered that the smaller beneficial effects of organic acids on growth might be due to buffering action, which would be particularly effective in medium III against the acid end products (e.g. gluconic acid) formed from glucose. Whilst organic acid cations, as buffers, may assist growth, the stimulations produced by them cannot be ascribed only to that cause. For example, in medium III, citrate, fumarate or acetate exerted appreciable buffering action but failed to stimu- 
late the growth of D3. The same is true for acetate and succinate with A1. Similar examples could be selected from the other test organisms, and it is concluded that the stimulation of growth produced by organic acids was due primarily to their participation in metabolism.

The pH values also showed that $(a)$ the metabolism of organic acids in medium II, and of lactate in medium IV, by lactaphilic strains produced more alkaline products; $(b)$ glucose was metabolized to acid end products by all strains, in many cases even when appreciable growth had not occurred (e.g. D3 in medium IV).

\section{Nitrogen requirements}

The tests designed to determine the carbon and energy requirements also give information concerning the nitrogen requirements of test organisms. All strains grew well on CAH and the ability to utilize ammonia as sole source of nitrogen has been discussed above (media III and IV).

\section{Table 3. Amino acids as source of nitrogen for the growth of Acetobacter strains}

Growth tests were made in defined glucose + lactate + salts medium (series A) or glucose $+0.2 \%\left(\mathrm{NH}_{4}\right)_{2} \mathrm{SO}_{4}$ + salts medium (series B). Growth is recorded as optical density values $\times 100$ after 7 days of incubation at $28^{\circ}$. Concentrations (g. $/ 100 \mathrm{ml}$.) of substrates were: series $\mathrm{A}$, amino acids, $0.6 ;\left(\mathrm{NH}_{4}\right)_{2} \mathrm{SO}_{4}, 0.3$ : series $\mathrm{B}$, DL-alanine, 0.72; other amino acids and $\mathrm{CAH}, 1 \cdot 2$.

\section{Strain}

A. acidum-mucosum
A. mobile
A. suboxydans $\mathbf{\gamma} 069$
A. ascendens
A. oxydans

A. capsulatum

A, gluconicum

A. turbidans

A. viscosum

$\begin{array}{cr}\begin{array}{c}\text { No added } \\ \text { nitrogen }\end{array} & \begin{array}{c}\text { L-Glutami } \\ \text { acid }\end{array} \\ & \\ 1 & 84 \\ 1 & 120 \\ 1 & 145 \\ 2 & 118 \\ 2 & 116\end{array}$

Serjes B
$\mathbf{6 3}$
$\mathbf{4 1}$
$\mathbf{3 9}$
$\mathbf{5 1}$

$\begin{array}{lc}\text { utamic } & \text { L-Aspartic } \\ \text { acid } & \text { acid }\end{array}$

\section{Series A}

\section{Growth on}

DL-Alanine L-Proline $\left(\mathrm{NH}_{4}\right)_{2} \mathrm{SO}_{4}$

CAH

$\begin{array}{rrr}102 & 114 & 150 \\ 92 & 21 & 152 \\ 155 & 126 & 170 \\ 123 & 8 & 165 \\ 95 & 5 & 154\end{array}$

$\begin{aligned} 82 & - \\ 28 & - \\ 130 & - \\ 4 & - \\ 3 & -\end{aligned}$

Single amino acids as sources of nitrogen. Preliminary studies by paper chromatography indicated that glutamate, proline, aspartate and $\alpha$-alanine were the amino acids most rapidly assimilated by acetic acid bacteria, particularly by lactaphilic strains, during growth on media containing CAH. Further tests on five lactaphilic strains grown on medium I, the CAH and ammonium sulphate of which was replaced by single amino acids, showed that good growth was obtained on L-proline, L-glutamate or L-aspartate, and sometimes on DL-alanine. The other 14 amino acids tested supported little or no growth, with few exceptions (e.g. L-arginine for Acetobacter mobile and L-leucine for $A$. oxydans). Similar tests on glycophilic strains, but in which $0.2 \%(\mathrm{w} / \mathrm{v})\left(\mathrm{NH}_{4}\right)_{2} \mathrm{SO}_{4}$ was included in the basal medium, showed that proline, 
glutamate and aspartate permitted amounts of growth of the order of those produced by CAH (Table 3). These tests indicated that L-glutamic acid and the amino acids biochemically related to it were key nitrogen compounds in the nutrition of acetic acid bacteria.

\section{Incubation of suspensions of organisms with amino acids}

When thick, non-proliferating suspensions of all test strains were incubated with solutions of proline, glutamate and aspartate, and the super-natant fluids were examined by paper chromatography, a clear difference between lactaphilic and glycophilic strains was observed as follows.

(a) No new ninhydrin-reacting material appeared in suspensions of glycophilic strains either in the absence of added supplements or in the presence of lactate, glucose, glucose + lactate or, in one case tested (Acetobacter capsulatum) in the presence of the full complement of growth factors as included in medium $I$.

(b) With all lactaphilic strains, new ninhydrin-reacting material, not detected in the control suspensions, appeared. The chief of these new materials in the proline, glutamate and aspartate systems possessed the chromatographic mobilities respectively of glutamate, aspartate and alanine.

Neither lactaphilic nor glycophilic strains yielded new ninhydrin-reacting materials when suspended in solutions of $\alpha$-alanine.

\section{Requirements for added growth factors}

The growth factor requirements of the strains hitherto unexamined in this respect were determined as described by Rainbow \& Mitson (1958). The results showed that all strains already classified as lactaphilic had no requirement for added growth factors. By contrast, all glycophilic strains required added pantothenate and nicotinate; strain $\mathbf{A 3}$ also required $p$-aminobenzoate for growth. The pattern of growth factor requirements found by Rainbow \& Mitson (1958) was therefore confirmed, and its relevance extended, to a further 21 strains of Acetobacter spp. The requirement of $A$. gluconicum for an additional unidentified growth factor (noted in the earlier work) was satisfied by uracil, which was not replaceable by cytosine, thymine or orotic acid. In the absence of uracil, $A$. gluconicum grew only after a lag of nearly 7 days, but organisms from such cultures, on transfer to uracil-free medium I, grew almost as well as in the same medium containing uracil, suggesting that selection for uracil-independence had occurred.

Note on p-aminobenzoate requirements. Neither pteroylglumatic acid nor folinic acid SF replaced $p$-aminobenzoate for the growth of Acetobacter capsulatum, $A$. gluconicum, $A$. turbidans and $A$. viscosum. However, the concentration of $p$-aminobenzoate needed to produce half-maximum growth of these strains was decreased by adding purines (of which hypoxanthine was the most effective) and, except with $A$. gluconicum, by vitamin $\mathrm{B}_{12}$. The $p$-aminobenzoate-sparing action of vitamin $\mathrm{B}_{12}$ was particularly great with $A$. capsulatum, the concentration of $p$-aminobenzoate needed for half maximum growth being decreased from 13 to $5 \mathrm{~m} \mu \mathrm{g} . / \mathrm{ml}$. by the presence of $0.015 \mathrm{~m} \mu \mathrm{g} . \mathrm{B}_{12} / \mathrm{ml}$. 


\section{DISCUSSION}

The present work provides further evidence that acetic acid bacteria may be divided into the two groups suggested by Rainbow \& Mitson (1958). The essential points of difference between the groups consist in the ability of lactaphilic, but not of glycophilic, strains: (a) to oxidize lactate and acetate; (b) to yield new ninhydrin-reacting substances when cell suspensions are incubated with proline, glutamate and aspartate; (c) to grow in media devoid of added pantothenate and nicotinate.

Our division of Acetobacter strains may be compared with that of Vaughn (1942). Vaughn's group 1 (oxidize acetic acid) and group 2 (do not oxidize acetic acid) correspond respectively to our lactaphilic and glycophilic groups. However, Vaughn's subdivision on the criterion of utilization of ammonia as the source of nitrogen seems less satisfactory since all our strains did so when other requirements for growth were also supplied. As a point of detail, it must also be noted that Vaughn's Acetobacter suboxydans should fit into our glycophilic group, whereas the three (typed) strains bearing this name which were examined by us were certainly lactaphilic.

Leifson (1954) suggested the recognition of two genera of acetic acid bacteria, retaining the generic name Acetobacter (type species $A$, aceti) for Vaughn's group 1. These are non-flagellated or peritrichously flagellated and oxidize acetate and/or lactate. For species which do not oxidize lactate or acetate (Vaughn's group 2) a new genus Acetomonas (type species Acetomonas suboxydans) was suggested by Leifson, the members of which are either non-flagellated or have polar multitrichous flagella. Again, Leifson's genera correspond closely to our groups, his proposed genus Acetomonas corresponding to our glycophilic strains. Thus, our work provides nutritional and biochemical evidence in support of the groups of acetic acid bacteria observed by Vaughn and by Leifson. However, whether the several criteria, which differentiate these groups, are of sufficient evolutionary importance to merit the separation of a genus Acetomonas from that of Acetobacter, is still an open question.

We are indebted to the Department of Scientific and Industrial Research for a grant to one of us (G. D. B.).

\section{REFERENCES}

Chamberlain, N. \& Rainbow, C. (1954). The formation of diazotizable amine and hypoxanthine by a yeast: possible implications in the biosynthesis of purines. J. gen. Microbiol. 11, 180.

Leifson, E. (1954). The flagellation and taxonomy of species of Acetobacter. Leeurvenhoek ned. Tijdschr. 20, 102.

Moore, W. B. \& Rainbow, C. (1955). Nutritional requirements and biochemical activities of brewery lactobacilli. J. gen. Microbiol. 13, 190.

Rainbow, C. \& Mrtson, G. W. (1953). Nutritional requirements of acetic acid bacteria. J. gen. Microbiol. 9, 871.

Vaughn, R. H. (1942). The acetic acid bacteria. Wallerstein Labs Commun. 5, 5.

(Received 3 January 1956) 